\title{
Inverse Laplace Transform of Some Rational Functions
}

\author{
R. WituŁA*
}

Institute of Mathematics, Silesian University of Technology, Kaszubska 23, 44-100 Gliwice, Poland

In this paper we propose a formula of recurrent nature for calculating the inverse Laplace transforms of some rational functions. The procedure and formulae enabling to determine the values of coefficients of obtained series are presented. Proposed procedure does not require any partial fraction decomposition. Moreover, it is proved that the radius of convergence of the received series (for the respective objective function) is equal to infinity. The proposed formula can find applications in wave optics and acoustics.

PACS: 02.30.Zz, 42.30.Lr, 42.25.-p, 42.65.Wi

\section{Introduction}

Scope of this paper is a description of the procedure of calculating the inverse Laplace transforms of the following rational functions:

$$
\frac{p(z)}{\left(z-z_{0}\right)^{n}+A\left(z-z_{0}\right)+B},
$$

where $A, B, z_{0} \in C, A B \neq 0, p \in \mathbf{C}[z], \operatorname{deg} p<n$ and all complex zeros of the trinomial $z^{n}+A z+B$ are simple.

Extraordinary in the proposed below formula (14) for the inverse transform of the original (1) is the fact that it contains only the coefficients of the polynomials from numerator and denominator of (1) (more precisely, it is dependent only on $A, B$ and coefficients of $p(z))$. The roots of this polynomials implicitly participate only in generating formula (14), therefore these roots do not appear in this formula at all. The procedure and formulae enabling to determine the values of coefficients of the received series are presented. For evaluating these formulae only the fundamental theorem of algebra appeared to be necessary. Moreover, it is proved that the radius of convergence of the received series (for the respective objective function) is equal to infinity.

\section{Example}

Let us start our deliberations with designating the inverse Laplace transform of the following rational function:

$$
\left(z^{8}+8 z+1\right)^{-1}
$$

Let us assume that $\xi_{j} \in \mathbf{C}, j=1,2, \ldots, 8$, are all complex roots of the polynomial $p(z):=z^{8}+8 z+1$. Because $p^{\prime}(z):=8\left(z^{7}+1\right)$, so

$$
p^{\prime}(z)=0 \Leftrightarrow z \in \sqrt[7]{-1} \Rightarrow|z|=1 \Rightarrow p(z) \neq 0,
$$

therefore all zeros of $p(z)$ are simple.

Let us set

$$
S_{k}:=\sum_{j=1}^{8} \xi_{j}^{k} \quad \text { and } \quad T_{k}:=\sum_{j=1}^{8} \frac{\xi_{j}^{k}}{1+\xi_{j}^{7}},
$$

for $k=0,1,2, \ldots$

\footnotetext{
* e-mail: Roman.Witula@polsl.pl
}

From the Newton-Girard formulae for polynomial $p(z)$ (see $[1,2])$ we obtain

$$
\left\{\begin{array}{l}
S_{0}=8, \quad S_{1}=\ldots=S_{6}=0, \quad S_{7}=-56, \\
S_{k+8}=-8 S_{k+1}-S_{k}, \quad k=0,1,2, \ldots
\end{array}\right.
$$

By induction we get

$$
\left|S_{k}\right| \leq\left\{\begin{array}{l}
7 \times 2^{k-4} \quad \text { whenever } \quad k \in 2 \mathbf{N}-1, \\
2^{k-5} \quad \text { whenever } \quad k \in 2 \mathbf{N} .
\end{array}\right.
$$

Moreover, for every $j \in\{1,2, \ldots, 8\}$ the following relations can be deduced:

$$
\xi_{j}\left(1+\xi_{j}^{7}\right)=\xi_{j}^{8}+\xi_{j}=p\left(\xi_{j}\right)-7 \xi_{j}-1=-7 \xi_{j}-1
$$

and

$$
\left(7 \xi_{j}+1\right)\left(8+\sum_{r=0}^{7}(-7)^{-r} \xi_{j}^{7-r}\right)=1-\frac{1}{7^{7}} .
$$

Hence we get

$$
\begin{aligned}
& -\left(1-\frac{1}{7^{7}}\right) T_{k}=\sum_{j=1}^{8} \xi_{j}^{k+1}\left(8+\sum_{r=0}^{7}(-7)^{-r} \xi_{j}^{7-r}\right) \\
& =\left(8-\frac{1}{7^{7}}\right) S_{k+1}+\frac{1}{7^{6}} S_{k+2} \\
& \quad-\frac{1}{7^{5}} S_{k+3}+\ldots-\frac{1}{7} S_{k+7}+S_{k+8}
\end{aligned}
$$

and, by (4) and (6), we obtain

$$
\left\{\begin{array}{l}
T_{0}=T_{1}=\ldots=T_{6}=0, \quad T_{7}=8, \\
T_{k+8}=-8 T_{k+1}-T_{k}, \quad k=0,1,2, \ldots
\end{array}\right.
$$

It is clear that all $T_{k}$ are integers. Furthermore, relation (5) is the Binet formula for $T_{k}$. Let us also observe that $\left|T_{k}\right| \leq 2^{k-16}$ for $k=22,23, \ldots$.

In the next step we can attempt to designate the inverse transform of function (2). We have the following partial fractions decomposition:

$$
\frac{1}{p(z)}=\sum_{j=1}^{8} \frac{1}{8\left(\xi_{j}^{7}+1\right)\left(z-\xi_{j}\right)} .
$$

Hence, there can be deduced the following formula:

$$
L^{-1}\left(\frac{1}{z^{8}+8 z+1}\right)(t)=\frac{1}{8} \sum_{j=1}^{8} \frac{\mathrm{e}^{\xi_{j} t}}{\xi_{j}^{7}+1}
$$




$$
=\frac{1}{8} \sum_{k=0}^{\infty} \frac{t^{k}}{k !} \sum_{j=1}^{8} \frac{\xi_{j}^{k}}{\xi_{j}^{7}+1}
$$

i.e.

$$
L^{-1}\left(\frac{1}{z^{8}+8 z+1}\right)(t)=\frac{1}{8} \sum_{k=0}^{\infty} T_{k} \frac{t^{k}}{k !},
$$

where coefficients $T_{k}$ are described by recurrence formula (7).

Remark 1. We note that $p(z)$ has only two real roots $\xi_{1} \approx-1.327011995189$,

$\xi_{2} \approx-0.125000007451$.

The remaining roots $p(z)$ are

$$
\begin{aligned}
& \xi_{3}=\bar{\xi}_{4} \approx-0.8207308648+\mathrm{i} 1.0530843114, \\
& \xi_{5}=\bar{\xi}_{6} \approx 0.3170740585+\mathrm{i} 1.3130419243 \\
& \xi_{7}=\bar{\xi}_{8} \approx 1.2296628076+\mathrm{i} 0.5843226746 .
\end{aligned}
$$

\section{Generalization}

Procedure of determining the inverse transform of function described by formula (1) is similar to the procedure described in detail in the above example. First, by applying the translation of resulting function for the Laplace transform

$$
L\left[\mathrm{e}^{z_{0} t} f(t)\right](z)=L[f(t)]\left(z-z_{0}\right),
$$

where $f(t)$ is an objective function (see [3-5]), function (1) could be replaced by a simple one

$$
\frac{p(z)}{z^{n}+A z+B} \text {. }
$$

Let $\xi_{j} \in \mathbf{C}, j=1,2, \ldots, n$ be all complex roots of polynomial $q(z):=z^{n}+A z+B$. Let

$$
S_{k}:=\sum_{j=1}^{n} \xi_{j}^{k} \quad \text { and } \quad T_{k}:=\sum_{j=1}^{n} \frac{\xi_{j}^{k}}{A+n \xi_{j}^{n-1}},
$$

for $k=0,1,2, \ldots$ Then, by the Newton-Girard formulae for polynomial $q(z)$ we have

$$
\left\{\begin{array}{l}
S_{0}=n, \quad S_{1}=\ldots=S_{n-2}=0, \\
\quad S_{n-1}=-(n-1) A, \quad S_{n}=-n B, \\
S_{k+n}=-A S_{k}-B S_{k-1}, \quad k=1,2, \ldots,
\end{array}\right.
$$

whereas

$$
\begin{aligned}
-n B & {\left[\frac{A}{n}+\left(-C^{-1}\right)^{n-1}\right] T_{k} } \\
& =\sum_{j=1}^{n} \xi_{j}^{k+1}\left[A+\sum_{r=0}^{n-1}\left(-C^{-1}\right)^{r} \xi_{j}^{n-1-r}\right] \\
& =\left(A+\left(-C^{-1}\right)^{n-1}\right) S_{k+1}+\left(-C^{-1}\right)^{n-2} S_{k+2} \\
& +\ldots+\left(-C^{-1}\right) S_{k+n-1}+S_{k+n},
\end{aligned}
$$

where

$$
C=\frac{(n-1) A}{n B} .
$$

Hence, by (11) and (12), we obtain

$$
\begin{aligned}
& T_{0}=0, \\
& {\left[\frac{A}{n}+\left(-C^{-1}\right)^{n-1}\right] T_{1}=\left\{\begin{array}{c}
1+A C \text { for } n=3, \\
1 \text { for } n \geq 4,
\end{array}\right.} \\
& {\left[\frac{A}{n}+\left(-C^{-1}\right)^{n-1}\right] T_{2}=\left\{\begin{array}{c}
A+C^{-2}-C^{-1}-2 C \\
\text { for } n=3, \\
A C-C^{-1} \text { for } n=4, \\
-C^{-1} \text { for } n \geq 5,
\end{array}\right.}
\end{aligned}
$$

etc., up to deriving $T_{n}$; whereas, from (11) and (12) we get the following recurrence equation:

$$
T_{k+n}=-A T_{k+1}-B T_{k}, \quad k=1,2, \ldots
$$

We can now designate the inverse Laplace transform of function (9). Let us start from the following partial fractions decomposition:

$$
\frac{p(z)}{q(z)}=\sum_{j=1}^{n} \frac{\xi_{j} p\left(\xi_{j}\right)}{q^{\prime}\left(\xi_{j}\right)\left(z-\xi_{j}\right)} .
$$

Hence, the formula written below may be derived

$$
\begin{aligned}
L^{-1} & {\left[\frac{p(z)}{q(z)}\right](t)=\sum_{j=1}^{n} \frac{p\left(\xi_{j}\right)}{q^{\prime}\left(\xi_{j}\right)} \mathrm{e}^{\xi_{j} t} } \\
& =\sum_{j=1}^{n} \frac{\xi_{j} p\left(\xi_{j}\right)}{-B n\left(1+C \xi_{j}\right)} \mathrm{e}^{\xi_{j} t} \\
& =\sum_{k=0}^{\infty} \frac{t^{k}}{k !} \sum_{j=1}^{n} \frac{\xi_{j}^{k+1} p\left(\xi_{j}\right)}{-B n\left(1+C \xi_{j}\right)} \\
& =\frac{-1}{B n\left[\frac{A}{n}+(-C)^{-n+1}\right]} \\
& \times \sum_{k=0}^{\infty} \frac{t^{k}}{k !} \sum_{j=1}^{n} \xi_{j}^{k+1} p\left(\xi_{j}\right)\left[A+\sum_{r=0}^{n-1}(-C)^{-r} \xi_{j}^{n-1-r}\right] \\
= & \frac{-1}{B n\left[\frac{A}{n}+(-C)^{-n+1}\right]} \sum_{k=0}^{\infty} \frac{t^{k}}{k !} \sum_{s=0}^{N} \alpha_{s} S_{k+1+s},
\end{aligned}
$$

where coefficients $\alpha_{s}$ are determined by the relation

$$
p(z)\left[A+\sum_{r=0}^{n-1}(-C)^{-r} z^{n-1-r}\right]:=\sum_{s=0}^{N} \alpha_{s} z^{s}
$$

and $N:=n-1+\operatorname{deg} p$. Furthermore, we have

$$
\sum_{j=1}^{n} \frac{\xi_{j}^{k+1} p\left(\xi_{j}\right)}{-B n\left(1+C \xi_{j}\right)}=\sum_{s=0}^{M} \beta_{s} T_{k+s},
$$

as long as $p(z)=\sum_{s=0}^{M} \beta_{s} z^{s}$ which, in consideration of (14), generates the following identity:

$$
-B n\left[\frac{A}{n}+(-C)^{-n+1}\right] \sum_{s=0}^{M} \beta_{s} T_{k+s}=\sum_{s=0}^{N} \alpha_{s} S_{k+1+s}
$$

At the end of this brief discussion we would like to notice two facts concerning the power series and recurrence equations discussed in this paper. First theorem is re- 
lated to the radius of convergence of series (14). Second theorem enables to state, in some cases, when the recurrence sequence $\left\{S_{n}\right\}_{n=0}^{\infty}$ (formula (11)) is bounded.

Theorem 1. Let $\left\{T_{n}\right\}_{n=0}^{\infty} \subset \mathbf{C}$ be a nontrivial linear recurrence sequence. Then the power series $\sum_{n=0}^{\infty} T_{n} z^{n}$ has the positive radius of convergence. More precisely, if the sequence $\left\{T_{n}\right\}_{n=0}^{\infty}$ has the following Binet form:

$$
T_{n}=\alpha_{1}(n) z_{1}^{n}+\alpha_{2}(n) z_{2}^{n}+\alpha_{3}(n) z_{3}^{n}+\ldots+\alpha_{k}(n) z_{k}^{n},
$$

where $\alpha_{j}$ is a complex polynomial for every $j=1,2, \ldots, k$ and $\left|z_{1}\right| \geq\left|z_{2}\right| \geq\left|z_{3}\right| \geq \ldots \geq\left|z_{k}\right|$, then from the CauchyHadamard formula we deduce that value of the radius of convergence of the power series $\sum_{n=0}^{\infty} T_{n} z^{n}$ is equal to $\left|z_{1}\right|^{-1}$ (for the power series $\sum_{n=0}^{\infty} \frac{T_{n}}{n !} z^{n}$ the radius of convergence is equal to infinity).

Referring to the known Theorem presented in [2] (giving the necessary and sufficient condition that all the roots of given polynomial from $\mathbf{C}[z]$ lie inside of the unitary circle), it is worth to notice some special case of this Theorem and its connection with our trinomial $z^{n}+A z+B($ see $[6,7])$

Theorem 2. Suppose $\alpha_{j} \geq 0,1 \leq j \leq k$. All the complex roots of polynomial

$$
z^{k}-\alpha_{1} z^{k-1}-\ldots-\alpha_{k}
$$

have moduli smaller than 1 if and only if $\alpha_{1}+\ldots+\alpha_{k}<1$.

\section{Conclusion}

The formula and method of its generating, presented in this paper, were confronted, among others, with $[4,5$, $8-12$ ]. However, it seems like the formula is completely new and original.

\section{References}

[1] M.I. Cirnu, Am. Math. 117, 67 (2010).

[2] N.B. Conkwright, Introduction to the Theory of Equations, Blaisdell Publ. Com., New York 1965.

[3] B. Davies, Integral Transforms and Their Applications, Springer, New York 1985.

[4] R. Witula, D. Slota, A. Warzynski, J. Integer Sequenc. 9, 1 (2006).

[5] M.L. Krasnov, A.I. Kiselev, G.I. Makarenko, Functions of a Complex Variable, Operational Calculus, and Stability Theory, series Problems and Exercises, Mir Publ., Moscow 1984.

[6] M. Kucharzewski, M. Kuczma, Scientific Journal of The Higher School of Pedagogy, Katowice, Section of Mathematics 3, 61 (1962) (in Polish).

[7] A.M. Cohen, Numerical Methods for Laplace Transform Inversion, Springer, New York 2007.

[8] B. Pustelny, T. Pustelny, Acta Phys. Pol. A 116, 383 (2009).

[9] J. Ignac-Nowicka, T. Pustelny, Z. Opilski, E. Maciak, W. Jakubik, M. Urbanczyk, Opt. Eng. 42, 2978 (2003).

[10] L. Debnath, D. Bhatta, Integral Transforms and Their Applications, Chapman \& Hall/CRC, Boca Raton 2007.

[11] T. Pustelny, J. Ignac-Nowicka, B. Jarzabek, A. Burian, Opt. Appl. 34, 551 (2004).

[12] R. Wituła, D. Slota, Appl. Math. Commun. 202, 348 (2008). 